\title{
Analysis of the Applicability of Electroactive Polymers in the Construction of Dispensing Valves
}

\author{
Damian KĄDZIELAWA ${ }^{\mathrm{a}, 1}$ and Wojciech SKARKA ${ }^{\mathrm{b}, 2}$ \\ a Sanhua Aweco Appliance Sp. z. o. o. Sp. K., Poland \\ bilesian University of Technology, Mechanical Engineering Faculty, Poland
}

\begin{abstract}
Technology of electroactive polymers which react by changing their geometric form under the influence of electrical voltage, has been developed for several decades. The main objective of the research carried out so far, was to develop a technology enabling the usage of such materials as an microscale valves activators. The developed solutions were dedicated mainly to the medical industry, in order to create very small applications, i.e. precisely dosing drugs. This article concerns the analysis carried out in the field of existing electroactive polymers. The conducted research focuses on the verification of their properties, in the context of the possibility to transfer and apply to the valves used in the household appliances. The detailed analysis will be conducted for confirmation, that current technology of electroactive polymers combined with a dedicated mechanical system of the prototype valve, will create favorable conditions for constructing a solution comparable with products present on the market. It will be crucial to confirm that the combination of material and mechanical aspects will ensure stable parameters of linear displacement, force, repeatability in the activation-deactivation cycle and number of cycles possible to reach. Chosen activator material must be examined to ensure smooth movement between the state of activation and precise return to the base shape. In addition, the analysis should indicate what is the environmental influence to the application, and how it will affect on the achievable parameters. The results of the research will allow to find an economically justified alternative to the currently used technology.
\end{abstract}

Keywords. Electroactive Polymer, Household Appliances, Prototype Valve

\section{Introduction}

The area of solutions for the white household appliances lost its momentum towards the end of the last decade of the twentieth century. In most cases the industry, and thus the companies dealing with providing the solutions for white household appliances, focused primarily on improving solutions developed over the previous decades, while the work on the implementation of new technologies and materials likely to improve this product segment has been discontinued. It is not a matter of stopping the work completely, however, a considerable slowdown is noticeable and the proposed development directions are usually based just on the improvement of existing research instead of an

\footnotetext{
${ }^{1}$ Corresponding Author, Email: damian.kadzielawa@polsl.pl.

${ }^{2}$ Corresponding Author, Email: wojciech.skarka@polsl.pl.
} 
attempt to introduce a market revolution. Only last year there was cautious try to resume activity and start to work on new directions of advancements in this area.

A highly specialized and a bit closed White Household industry requires at the moment a fresh look at the overall technology developed over the past years and an attempt to implement new ideas in the material and mechanical areas. Such approach can directly affect the creation of new, economically justified solutions providing novelty and innovation to the market. Thanks to this, in addition to reducing the costs of the already used solutions, we can impact their attractiveness and the usefulness for end users.

The liquid flow management technology that exists today on the market is mainly based on electromagnetic coils. Although this solution is resistant to difficult working conditions such as high humidity, temperature of the working environment, required high amounts of cycles during the product life and aggressive chemicals used in the systems, it is not ideal and introduce a large spectrum of risk. In the series production of an electromagnetic coil, which is a component of flow control valves in white household appliances, they are exposed to many undesirable occurrences: the diversity of the electromagnetic field needed for activation, the system wear affecting the efficiency, the aggressive environment having a direct impact on the lifetime of the technology, electric shortcuts and risk of electric shock, aging of the material, generated unwanted noise during activation and the price of copper needed to produce the activator. These issues show a great demand for alternative solutions - based on new material technologies and their direct advantages.

As a consequence, it seems reasonable to try to develop new concepts, allowing in a simple and effective way to overcome the previously mentioned negative effects of current solutions. This is an area with a high potential to introduce further changes such as reducing the dimensions of these devices, working on increasing efficiency and durability, improving work ergonomics and resistance to the impact of conditions coming directly from the environment in which they are dedicated to operate. It can bring high benefits to both producers of white household appliances and their end users.

Ofcourse there are alternative solutions like i. e. wax motors. Unfortunately they are not so flexible as solenoid actuators, works very slow and due to now, as literature shows, there was no attempts to overcome usage of standard coil with cheap polymer solution.

\section{Valves with electromagnetic solenoid actuator. Specification and parameters}

The majority of activators currently used on the white household appliances market are based on solutions that are derived from electromagnetic activator technology. They have many advantages but they also have a number of significant disadvantages in parallel. Among the disadvantages we can definitely distinguish the nonhomogeneous electromagnetic field and the relatively high production cost, due to the fact the material responsible for generating the mentioned field is a coil made from a copper wire. In addition, AC activation has a direct effect on the core oscillation tendency, and thus on undesirable acoustic effects.

By definition, the essence of the operation of such activation system is very simple. It is based on two states of the valve system: opened and closed. This simple feature allows you to easily and efficiently perform most of the required flow management tasks on your device. The liquid is currently flowing or completely blocked. The further part of the process is already directly defined by the valve type that has been used. It defines what and how the medium should work during the device's operating cycle to achieve 
the assumed goals: opening / closing the flow, changing direction or redirecting it to another circuit.

The most popular and most commonly used types of valves controlled by an electromagnetic activator due to the operating parameters are:

- Due to the flow opening / closing element: direct-acting valves, intermediate operating valves

- Due to the construction: $2 / 2$ valves, $3 / 2$ valves, $4 / 2$ valves, $5 / 2$ valves Item

- Due to the initial state: normally open, normally closed

The valve type defines in a direct way which flow control functions are possible to achieve in the system. The most important parameters of the valves are primarily the linear displacement that can be achieved during the activation, the force with which the activator acts on the system, the repeatability of the process depending on the number of cycles, as well as the transition speed from 0 to 1 and back.

Taking into account the above requirements, linear displacements of a few to several millimeters should be considered as a reference. The forces achieved during the activation process are usually below $5 \mathrm{~N}$. Unfortunately, the mentioned force parameter is directly dependent on the resistance and the associated temperature of the system when the activation time increases, the activator temperature raises. The higher temperature affects the decrease of achievable activation force and increase of current. In addition, if for some reason the resistance in the system increases (caused by system wear, working conditions) and we will come closer to the limit of the force possible to achieve in the system during activation in opposite to the spring force responsible for returning to the initial state of the system, we will get an oscillation effect. As a consequence, the risk of the core oscillation increases, which results in a very unpleasant acoustic effect. This is the case when the system is supplied with AC current. This is a side effect that is highly undesirable by manufacturers of complete white household appliances.

The above-described disadvantages of systems based on electromagnetic activators led to an attempt to find and develop alternative methods that could be used in similar applications of the white household appliances industry without losing any functional requirements. In a first row we should be able to completely remove negative oscilation effect using electroactive polymer based solution. Only question mark which still exist is to find way to create solution with stable displacement and force parameters. This is area where the main focus will be putted during further research.

\section{Electroactive polymers and their properties. Parameters possible to be achieved}

Recent research shows the electroactive polymers became the alternative for present solutions. Considering a number of studies and publications, these materials show great potential. Available studies even speak of the possibility of achieving an elongation of $12 \%$ [1] and the strength of several mega pascals [2]. Until now, electroactive polymer materials have been considered mainly as micro-scale activators for use in medical solutions such as, for example, automatic drug dispensers in the medical industry [3]. The area of solutions for white household appliances is associated with much larger dimensions, which allows a slightly different view on the attempt to create a solution 
based on an activator of polymer material. More space in application gives more flexibility during concepts building.

In addition, a great advantage of the use of electroactive materials may be the ability to smoothly control the actuator state not only in the range of extreme open / closed values, but also everything that is between these states. This is definately an additional value. It brings possibilities to extend further resarch with possibility to look for the new iteresting functions or even trials to redefine how full system works.

Research carried out so far shows a great potential in the area of implementing the basic requirements for activators with the use of polymeric materials activated by electrical voltage [4] [5]. The achieved parameters of displacement and force during activation seem to be similar to current solutions, and the benefits of using such materials can significantly affect the elimination of negative aspects of the operation of standard electromagnetic activators in the form of undesirable noise. In addition, it seems to be a great advantage to use low voltages to achieve the activation state. Another important advantage may be the fact that we are talking about creating a material itself, being an alternative to an electromagnetic coil, that is a set of components enabling the implementation of the described function. Currently, it is a assembly consisting of a coil core, a wound of copper wire, a layer of plastic overmoulding which is a protection for wound coils and dedicated connector enabling the connection of the assembly to a complete system.

In the case of successful research and achievement of the assumed parameters, there is a chance to eliminate a number of components of the system: spool, wire and insulating layer protecting from electric shock. This can have a significant impact on reducing the costs needed to produce such activators. The following illustrations show the assumed operating scheme in two extreme states: activated and deactivated [Fig.1] [Fig.2].

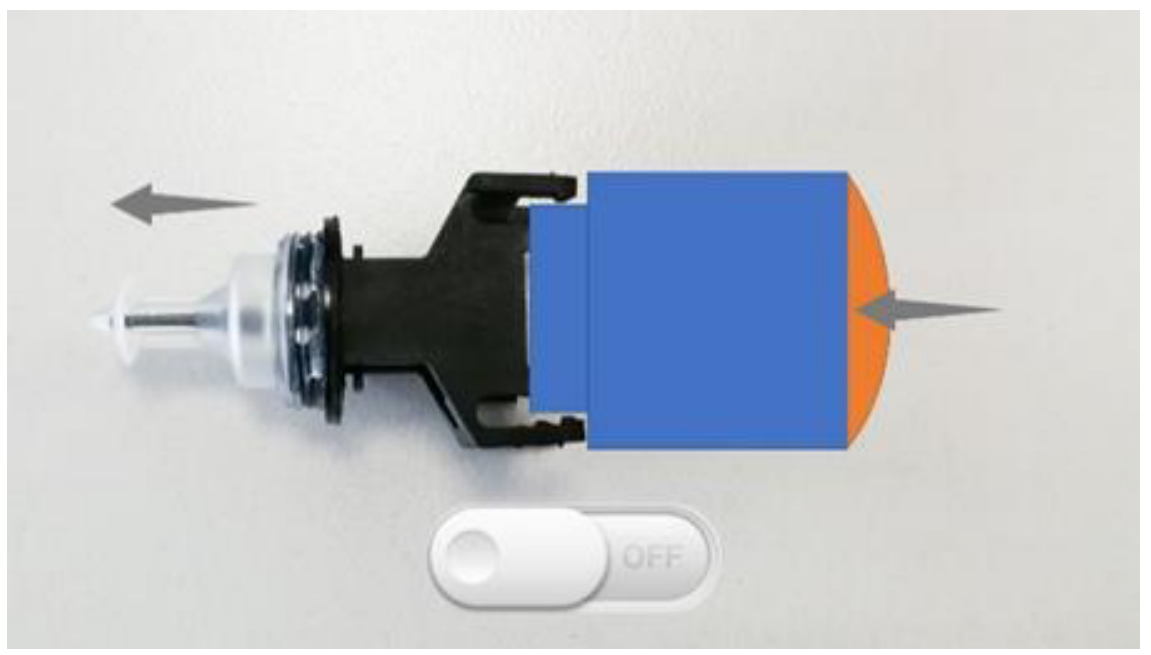

Figure 1. Deactivated electro active actuator - valve closed. 


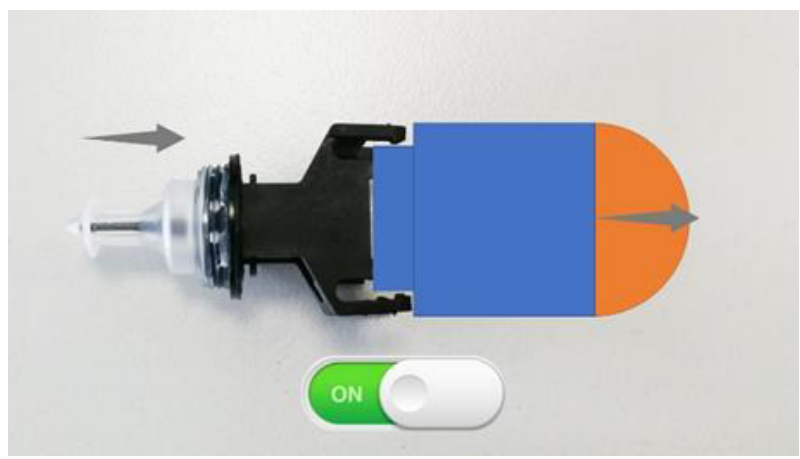

Figure 2. Activated electro active actuator - valve opened.

The size of manufactured household appliances also creates the possibility of connection with a dedicated mechanical system, which would allow the increase of the achieved parameters. In other words the use of available space and the use of a specially designed mechanical system that allows to strengthen the parameter of displacement and force.

\section{Subject introduction}

At the moment, conceptual work is underway to build an alternative system which is a combination of a mechanical direct-acting valve and an activator created from an electroactive polymer. As reference for the solution being built, one of the standard applications is shown in the picture below [Fig. 3].

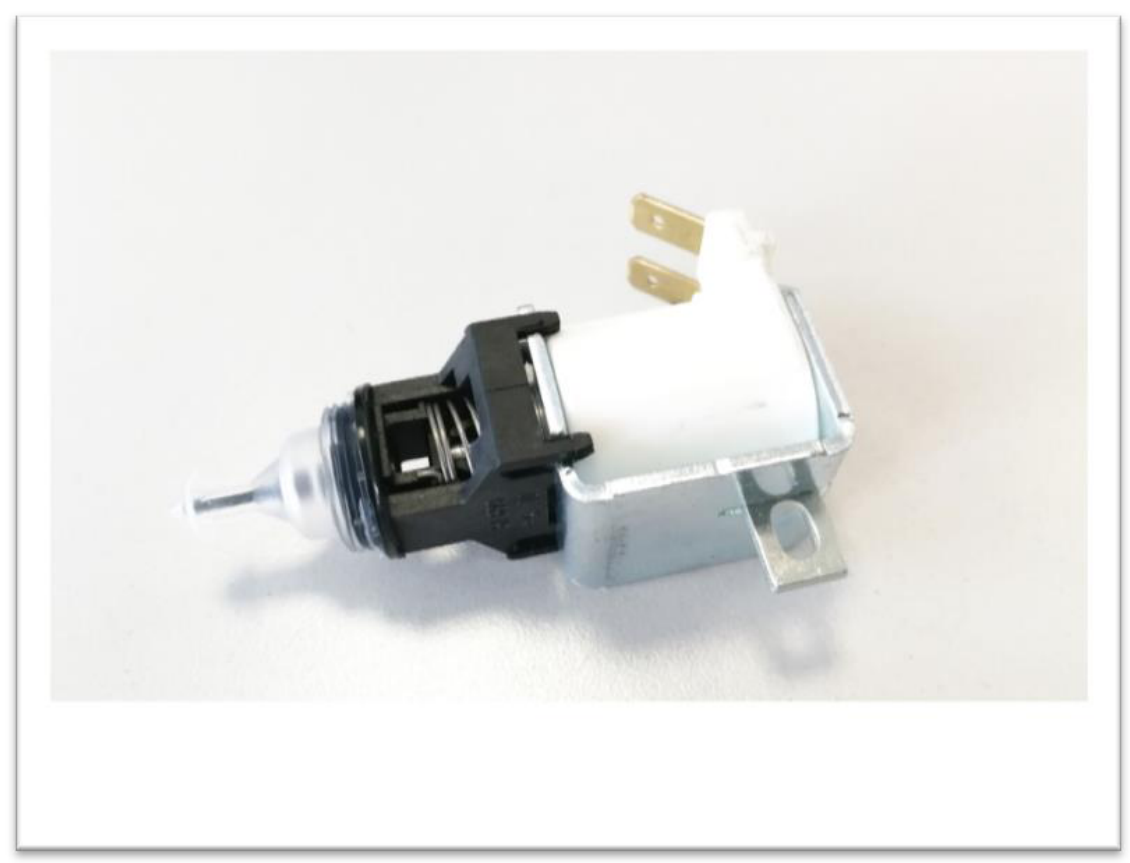

Figure 3. Standard solenoid actuated valve. 


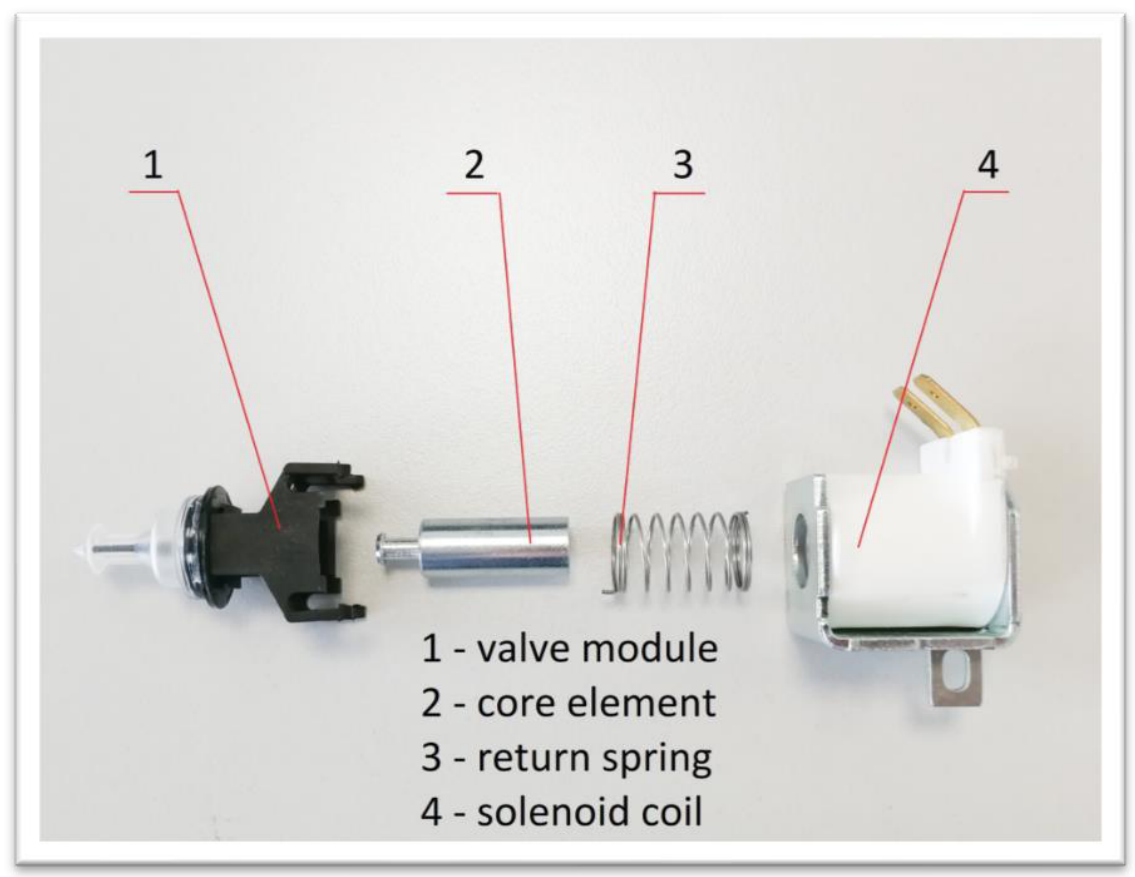

Figure 4. Exploded view of the reference valve system with an electromagnetic activator.

The assumption for the conducted works was to determine the reference parameters of the existing solutions and attempt to develop such a new valve construction to get as close as possible to the parameters of linear extension and activation force.

The selected valve system is shown in the figure below [Fig. 4]. It consists of an electromagnetic activator assembled form a coil (4), a steel fitting that integrates the whole system (4), a core responsible for the linear displacement of the valve (2), a spring (3), a silicon element and a plastic frame that is part of its support (1). The reference system shows a linear displacement of $6 \mathrm{~mm}$ and a maximum strength of $3.4 \mathrm{~N}$.

In the final solution, the entire element of the electromagnetic activator (coil) is to be replaced by an electrically activated polymeric material with a dedicated mechanical system connecting the linear movement of the actuator and the target opening of the valve. An overview of the new layout is shown in the picture below [Fig. 5]. It presents three important areas responsible for various functions of the complete valve system:

- $\quad$ The part marked with the letter [A] is an existing valve assembly mounted on a dedicated carrier enabling linear movement.

- Segment marked with the letter [B] is a part which will be dedicated to a special mechanism of linear displacement transfer of the electroactive activator on the target valve movement.

- The third part marked with the letter [C] is the area of the activator itself in the form of an electroactive polymeric material. 


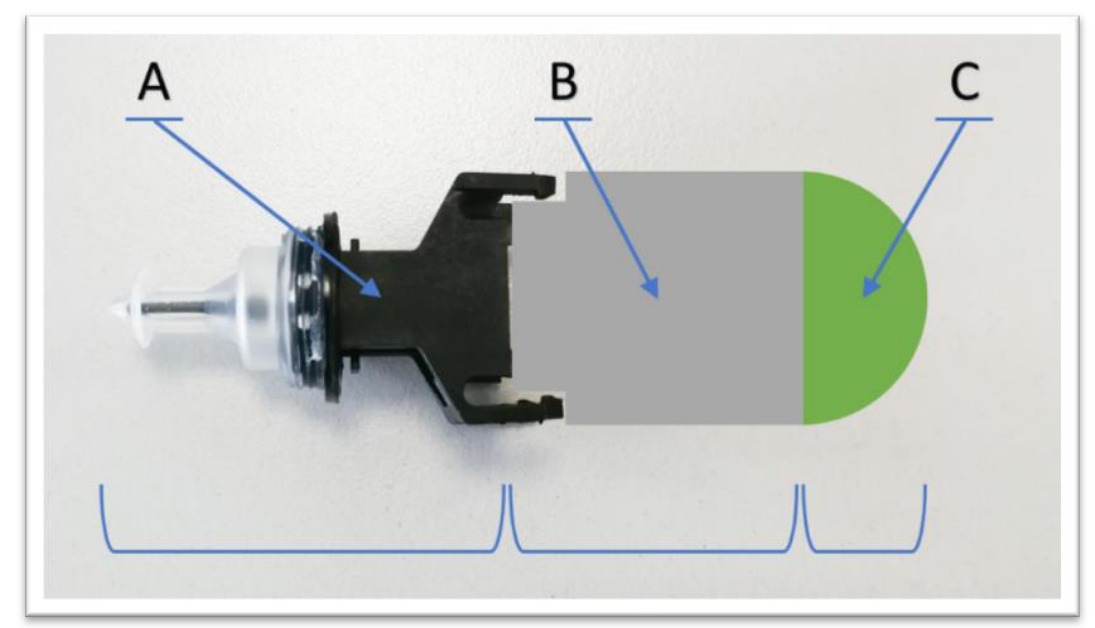

Figure 5. Diagram of a new valve system with activator made of electroactive polymer material.

The works performed so far have allowed to build the basic assumptions of the conducted research, choose constructed system's design and the verification methodology of the parameters achieved. At the same time, an attempt is made to select the optimal electroactive polymer material allowing for the implementation of the intended purpose and the method of its application. There are many possibilities such as the usage of gel -type material, the choice of a possible carrier, its shape and size. All of this is currently tested and thoroughly analyzed. Obtained results will allow to make a final decision and move to the next stage of the research.

\section{Alternative materials and concepts}

Due to the fact that work on the selection of the final solution is still underway, two other areas of alternatives are also considered: dielectric elastomers [6] and magnetorological elastomers [7].

The first of them requires much higher voltage to be activated than electroactive polymers, on level of several kilovolts. As a result, there might be a need for additional insulation due to the usage in a high humidity work environment and to maintain safe conditions of use. The second one - magnetorological elastomers - requires an electromagnetic field for activation, which means that only some elements of a traditional electromagnetic actuator can be eliminated. This will reduce the cost-effectiveness of the solution at the very beginning.

In order to obtain a wider perspective in the selection of the final material, the conducted research assumes a review of the full range of the possibilities and final multicriteria assessment, allowing to make a final decision.

It should be noted that a much wider field of potential solutions and a complete change in the approach to valve construction is being analyzed. This includes extending the scope of research to the material used to build a new activator. The idea is to develop a completely new, fresh and innovative approach to the design and operation of the valve. It is a methodology that directly breaks with the idea of a traditional valve, assuming a bold attempt to develop and implement a new technology. Unfortunately, due to the work on patent applications, the results of new research can not yet be presented. 


\section{Summary and further research}

Based on the data collected so far and according to the existing research and publications, the search direction seems to be well-founded. In addition, the first laboratory tests conducted on the materials themselves dedicated to the creation of future activators show very promising results: displacement, generated strength of activation and repeatability possible to achieve.

At the current stage of work, the parameters required for proper system operation and function retention are already known and fully defined. Advanced research on the construction of the numerical model is in progress. This will allow to determine the geometrical concept of the solution sought - work is carried out in close cooperation with one of the European universities, which specializes in numerical calculations. The created model will intensify the research at the laboratory stage.

At the same time, work is underway on the preparation of material samples that will be tested in the next stage. It was planned to choose the most promising material by multi-criteria evaluation.

The final solution may lead to the opening of a new chapter in the development of future valve applications in white household appliances. The final verification is conditioned by the results in the phase of full laboratory tests on selected materials combined with a dedicated mechanism of linear motion transfer after activation. The complete solution must be considered in the context of full implementation in the target valve system.

\section{Acknowledgement}

Publication partially supported as a part of the Rector's grant in the area of scientific research and development works. Silesian University of Technology, grant number 10/060/RGJ18/0082

\section{References}

[1] L. Bay, K. West, P. Sommer-Larsen, A Conducting Polymer Artificial Muscle with 12 \% Linear Strain; Advanced Material, 2003, 15, No.3, February 5, 310-313.

[2] W. Lu, E. Smela, P. Adams, G. Zucarello, B. R. Mattes, Development of Solid-in-Hollow Electrochemical Linear Actuators Using Highly Conductive Polyaniline; Chem. Mater, 2004, 16, pp. 1615-1621.

[3] H. Xu, C. Wang, C. Wang, J. Zoval, M. Madou, Polymer actuator valves toward controlled drug delivery application, Biosensors and Bioelecronics, 21 (2006) 20194-2099.

[4] H. Yan, K. Tomizawa, H. Ohno, N Toshima, All-Solid Actuator Consisting of Polyaniline Film and Solid Polymer Electrolyte, Macromolecular Materials and Engineering, 2003, 288, pp. 578-584.

[5] Patent No.: US6,685,442 B2; Actuator device utilizing a conductive polymer gel; United States Patent

[6] A. Nicolau-Kuklinska, P. Latko-Duralek, P. Nakonieczna, K. Dydek, A. Boczkowska, J. Grygorczuk, A new electroactive polymer based on carbon nanotubes and carbon grease as compliant electrodes for electroactive actuators; Journal of Intelligent Material Systems and Structures, Vol. 29, No. 7, 2018, pp. 1520-1530, https://doi.org/10.1177/1045389X17740979.

[7] A. Boczkowska, The role of microstructure in shaping the properties of intelligent magnetorheological composites (in Polish), Warsaw University of Technology Faculty of Materials Science, Warsaw, June 2010 . 